\title{
Sonda Metálica Reduzida para a Caracterização Térmica de Polímeros
}

\author{
Luís F. dos S. Carollo, Daniel A. Gravena, Sandro M. M. de L. e Silva \\ Laboratório de Transferência de Calor, UNIFEI
}

\begin{abstract}
Resumo: Vários métodos têm sido utilizados na determinação da condutividade e difusividade térmica. Dentre esses, cita-se o Método do Fio Quente, que é muito utilizado por ser um método transiente de troca de calor que determina estas propriedades de forma simultânea e rápida. Neste trabalho o Método do Fio Quente com uma sonda de dimensões reduzidas é usado para obter a condutividade e difusividade térmica do Policloreto de Vinila (PVC) e da Poliamida. Este método se baseia na passagem de uma corrente contínua através de um fio de resistência, gerando calor que aquece uma sonda inserida na amostra. Da dissipação do calor é possível calcular a condutividade térmica. Para determinar a difusividade térmica minimiza-se uma função erro, definida pelo quadrado da diferença entre a temperatura experimental e a numérica. A temperatura numérica é obtida pela solução da equação da difusão unidimensional em coordenadas cilíndricas utilizando o Método de Diferenças Finitas com formulação explícita. O diferencial deste trabalho refere-se às pequenas dimensões da sonda, possibilitando a utilização de amostras menores, além de apresentar um novo dispositivo para fixar corretamente os componentes. Os resultados estimados apresentaram boa concordância com a literatura.
\end{abstract}

Palavras-chave: Condutividade térmica, difusividade térmica, estimativa simultânea, condução de calor e otimização.

\section{Reduced Metallic Probe for the Thermal Characterization of Polymers}

\begin{abstract}
Several methods have been used to determine thermal conductivity and thermal diffusivity. Among these, the Hot Wire Method is widely used because it is a transient heat transfer method which allows the determination of these properties simultaneously and quickly. In this work, a reduced dimension metallic probe is used to obtain these properties for Polyvinyl Chloride (PVC) and Polyamide samples by using the Hot Wire Method. This method consists in passing a current through a resistance wire to generate heat, which increases the temperature of a probe inserted in a sample. From the heat dissipation it is possible to calculate the thermal conductivity. To determine the thermal diffusivity, optimization techniques are used to minimize an error function, defined by the square of the difference between the experimental and numerical temperatures. The numerical temperature is obtained by the solution of a one-dimensional diffusion equation in cylindrical coordinates, by using the Finite Difference Method with explicit formulation. The advantage of this study is the use of a small dimension probe allowing the use of samples with reduced dimensions. In addition it presents a new device to fasten the components into the probe. The estimated properties were in good agreement with the literature.
\end{abstract}

Keywords: Thermal conductivity, thermal diffusivity, simultaneous estimation, heat conduction and optimization.

\section{Introdução}

O desenvolvimento de novos materiais tem sido uma área de pesquisa intensa e tem recebido uma demanda tecnológica crescente. Pode-se citar, por exemplo, o uso destes materiais em aplicações de isolamento térmico, onde se tem a necessidade de minimizar a transferência de calor entre dois sistemas físicos ou onde é necessário o alcance da máxima eficiência da transferência de calor. Desta forma, decidiu-se analisar neste trabalho o Policloreto de Vinila (PVC) e a Poliamida, devido ao baixo custo, facilidade de aquisição no mercado, vasta aplicação em ambientes industriais e uso destes em processos de isolamento, principalmente o PVC. Além disso, esses materiais apresentam boa usinabilidade, característica que facilita a confecção do furo onde posteriormente a sonda será inserida. Entretanto, para o uso destes materiais em processos é necessário a determinação de sua condutividade térmica, $k$ e de sua difusividade térmica, $\alpha$. Atualmente existem inúmeros métodos para a determinação dessas propriedades, destacando-se três principais métodos utilizados. O Método Flash desenvolvido por Parker et al. ${ }^{[1]}$ que é empregado na determinação da difusividade térmica. Vários trabalhos fazem uso deste método, sendo citados Eriksson et al. ${ }^{[2]}$ e Santos et al. ${ }^{[3]}$. O Método da Placa Quente Protegida ${ }^{[4]}$, muito utilizado na determinação de $k$ de materiais isolantes, é considerado por inúmeros pesquisadores como Wulf et al. ${ }^{[5]}$ e Lima et al. ${ }^{[6]}$, entre outros, o método mais preciso e confiável. O Método do Fio Quente é um método absoluto, direto e não estacionário descrito primeiramente por Schieirmacher ${ }^{[7]}$. Sua primeira aplicação prática foi reportada por Van der Held e Van Drunen ${ }^{[8]}$, na determinação da condutividade térmica de líquidos.

Blackwell $^{[9]}$, utilizando uma variação deste método, desenvolveu um modelo matemático para a determinação de $k \mathrm{e}$ $\alpha$ de rochas em campo. Esta variação do Método do Fio Quente pôde ser realizada com a inserção de uma sonda cilíndrica, no centro axial da amostra que se deseja medir. A sonda tem a função de dissipar calor por efeito Joule e medir a temperatura no interior da amostra. Assim, $k$ é determinada através do transiente de temperatura gerado pela fonte de calor no material. Este método também pode ser empregado para a obtenção de $\alpha$, através da minimização de uma função objetivo. Porém é necessária a aplicação de um termopar na superfície da amostra, para verificar a condição de isolamento.

Haupin $^{[10]}$ utilizou pela primeira vez a técnica do fio quente na determinação de $k$ de materiais cerâmicos. Mais recentemente, Fujii et al. ${ }^{[11]}$ propôs um método chamado de "método do fio quente curto transiente", que pode ser usado para medir de $k$ e $\alpha$ de líquidos simultaneamente. Posteriormente, pôde-se medir estas propriedades de muitos tipos de líquidos, como polímeros derretidos (Zhang et al. ${ }^{[12]}$ ), carbonatos fundidos (Zhang et al. ${ }^{[13]}$ )

Autor para correspondência: Luís F. dos S. Carollo, Laboratório de Transferência de Calor, Instituto de Engenharia Mecânica, Universidade Federal de Itajubá - UNIFEI, Av. BPS, 1303, Pinheirinho, CP 50, CEP 37500-903, Itajubá, MG, Brasil, e-mail: felipecarollo@unifei.edu.br 
e refrigerantes alternativos (Tomimura et al. $^{[14]}$ ). Em constante atualização, outros autores propuseram mudanças com o intuito de determinar propriedades de outros materiais (Nahor et al. ${ }^{[15]}$ e Adjali e Laurent ${ }^{[16]}$ ).

Yamasue et al. ${ }^{[17]}$ desenvolveu o método do fio-quente não estacionário para medir as propriedades de metais fundidos, onde mercúrio e chumbo líquidos foram utilizados primeiramente. Posteriormente, a propriedade $k$ do silício e do germânio, ambos na fase líquida, foi determinada com sucesso.

Cheng e $\mathrm{Xie}^{[18]}$ apresentaram uma sonda fina para determinar a condutividade térmica de amostras como maçã, músculos, água destilada e etileno glicol. A desvantagem desse trabalho refere-se ao fato da sonda ter um diâmetro externo de $0,6 \mathrm{~mm}$, inviabilizando o processo de furação de qualquer amostra rígida.

Zhang et al. ${ }^{[19]}$ apresentaram o método do fio quente curto para determinar $k$ e $\alpha$ do ar. As propriedades puderam ser determinadas quando os dados numéricos, obtidos pela solução do modelo bidimensional, se tornavam iguais aos dados experimentais.

Dos trabalhos citados acima, é possível perceber que em alguns a determinação das propriedades ocorre para apenas uma propriedade, e em outros há a dificuldade em determinar as duas propriedades através de um mesmo experimento. Além disso, muitos trabalhos considerando o Método do Fio Quente utilizam sondas metálicas de grandes dimensões, o que acarreta na necessidade de analisar amostras com tamanhos elevados, dificultando tanto do ponto de vista econômico e prático, pois a bancada se torna cara e de difícil manuseio. Portanto, é proposto neste trabalho, utilizar o Método do Fio Quente com uma sonda metálica de pequenas dimensões para determinar $k$ e $\alpha$ de materiais poliméricos, através de um mesmo experimento. A condutividade térmica é calculada através do transiente de temperatura gerado por uma fonte de calor ideal, infinitamente longa e fina num meio material finito. Enquanto, a difusividade térmica é estimada através da minimização de uma função erro quadrática utilizando-se a técnica seqüencial de otimização BFGS (Broyden-Fletcher-Goldfarb-Shanno).

A grande contribuição deste trabalho refere-se as pequenas dimensões da sonda e ao dispositivo de fixação dos componentes internos da sonda, como o termopar e o fio de resistência. Esse diferencial garante maior flexibilidade a montagem, pois pode-se utilizar amostra menores, diminuindo o custo e a dificuldade de locomoção da bancada. Deve-se ressaltar que os resultados obtidos por essa técnica são satisfatórios quando comparados aos valores da literatura.

\section{Fundamentos Teóricos}

\section{Determinação da condutividade térmica, $k$}

A sonda linear é um instrumento simples, utilizada na determinação de $k$ e $\alpha$ de materiais isolantes. A sonda consiste em um tubo fino de metal que possui em seu interior uma resistência de aquecimento e um termopar, o qual mede a temperatura em função do tempo. O procedimento padrão de determinação das propriedades termofísicas é referenciado pela norma ASTM D5334-08 ${ }^{[20]}$, possuindo como base matemática o Método Clássico da Sonda Linear, apresentado por Blackwell ${ }^{[9]}$. A Figura 1 apresenta os detalhes da amostra a ser estudada.

O método supõe uma fonte linear, imersa em um meio finito (isotrópico e homogêneo) e com temperatura inicial igual a $T_{0}$. Assim, a equação da difusão em coordenadas cilíndricas para o modelo da Figura 1 é dada por:

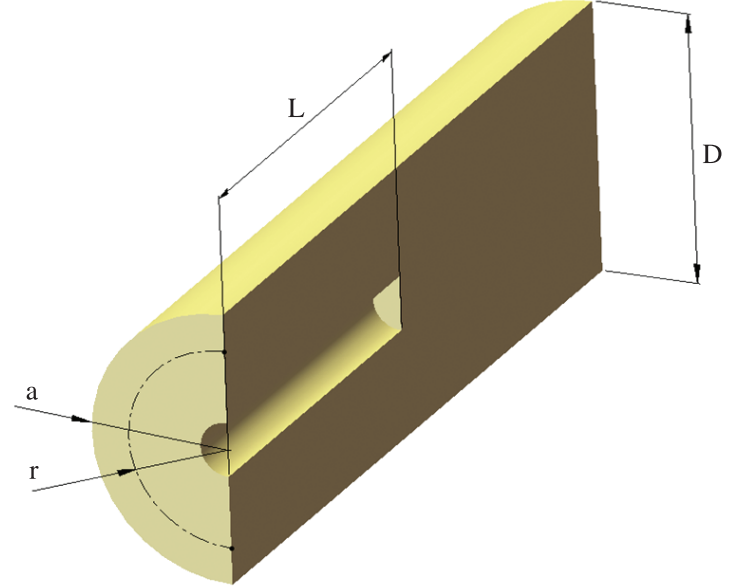

Figura 1. Detalhes da amostra.

$$
\frac{\partial^{2} T}{\partial r^{2}}+\frac{1}{r} \frac{\partial T}{\partial r}=\frac{1}{\alpha} \frac{\partial T}{\partial t} \quad 0<r<D / 2, t>0
$$

com a seguinte condição inicial Equação 2 e as condições de contorno, Equação 3 e Equação 4:

$$
\begin{array}{ll}
T=T_{0} & \text { para } r>0, t=0 \\
-2 \cdot \pi \cdot r \cdot k \frac{\partial T}{\partial r}=q=c t e & \text { para } r=a \\
\frac{\partial T}{\partial r}=0 & \text { para } r=D / 2
\end{array}
$$

onde: $\alpha$ e $k$ são, respectivamente, a difusividade e a condutividade térmica do material a ser analisado e $q$ é a potência dissipada por unidade de comprimento da fonte linear. A partir da solução da Equação 1, é possível concluir que em um gráfico de $\Delta T$ em função de $\ln (t)$, tem-se uma região linear cujo coeficiente angular é $\mathrm{m}=q / 4 \pi k$, para tempos longos. $\mathrm{O}$ detalhamento passo a passo dessa solução pode ser encontrado em Özisik ${ }^{[21]}$. Essa região linear usada para o cálculo da condutividade térmica sempre será a região intermediária do experimento, logo a condutividade térmica da amostra pode ser expressa na forma:

$$
k=\frac{q}{4 \pi m}
$$

A potência dissipada por unidade de comprimento da sonda, $q$, considerada constante, é dada por:

$$
q=\frac{R \cdot I^{2}}{L}
$$

onde $I$ é a corrente elétrica aplicada na sonda linear, $R$ é a resistência elétrica do fio no interior da sonda e $L$ é o comprimento do fio de resistência dentro da sonda.

\section{Determinação da difusividade térmica, $\alpha$}

Determinado $k$ pela Equação 5 obtém-se $\alpha$ minimizando a função objetivo baseada no erro quadrático entre a temperatura experimental e a numérica. A temperatura numérica é obtida através da solução da equação da difusão em coordenadas cilíndricas, utilizando o método de diferenças finitas com formulação explícita. Logo, o valor de $\alpha$ é o valor ótimo que minimiza a Equação 7:

$$
F=\sum_{i=1}^{N p t}\left[Y_{i}-T_{i}\right]^{2}
$$

onde $i$ é o contador de pontos, $N p t$ é o número total de pontos, $Y$ é a temperatura experimental e $T$ é a temperatura numérica. 
A minimização desta equação foi realizada utilizando a técnica seqüencial de otimização BFGS (Broyden-FletcherGoldfarb-Shanno) apresentada em Vanderplaats ${ }^{[22]}$. Esta técnica é uma particularidade dos Métodos da Métrica Variável. As vantagens deste método são a rápida velocidade de convergência e a facilidade para se trabalhar com inúmeras variáveis de projeto. Por se tratar de um método de primeira ordem é necessário conhecer o gradiente da função erro. Para o cálculo do gradiente é utilizado neste trabalho o pacote computacional Design Optimization Tools - DOT (Vanderplaats $^{[22]}$ ).

\section{Montagem Experimental}

\section{Descrição da sonda linear}

Apresenta-se na Figura 2, os detalhes da sonda linear de aço inox com 30,50 mm de comprimento e 1,27 mm de diâmetro. $O$ fato da sonda utilizada ser de pequenas dimensões é a grande vantagem deste trabalho, além de ser a tendência do desenvolvimento desta técnica como apresentado por Cheng e Xie ${ }^{[18]}$ e Zhang et al. ${ }^{[19]}$. A desvantagem de utilizar sondas com maior diâmetro é a necessidade de se utilizar amostras maiores, dificultando o manuseio e aumentando o custo da bancada. Outro diferencial do método utilizado é a confecção de um dispositivo que fixa os componentes inseridos na sonda, garantindo o posicionamento ideal dos mesmos e permitindo fácil manuseio de todo o aparato entre ensaios, demonstrado pela Figura 3. Esses componentes são o termopar que

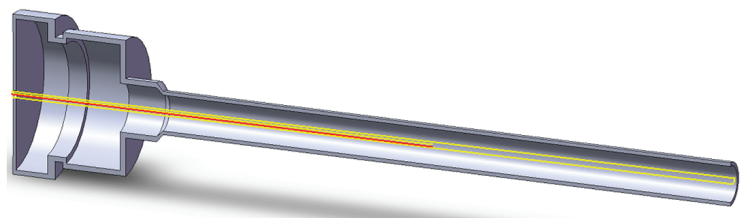

Figura 2. Detalhes da sonda.

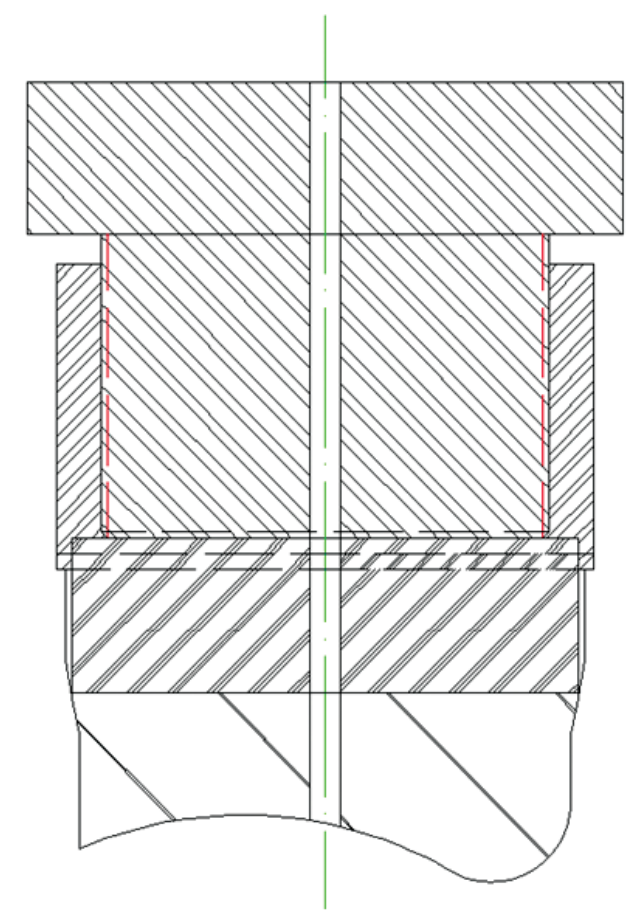

Figura 3. Dispositivo em corte longitudinal. deve estar localizado no meio da sonda e o fio de resistência que deve percorrer toda a extensão interior da sonda.

O método baseia-se na colocação de um termopar até o meio da sonda e um fio de resistência, que deve fazer o contorno interno da mesma. Passando-se uma corrente elétrica pelo fio de resistência, ocorre uma dissipação de calor que se propagará para o material, um meio finito, gerando um campo transiente de temperaturas. Para minimizar o problema de resistência de contato entre a parede interna da sonda e os componentes, utiliza-se pasta térmica de prata (Arctic Silver 5) para preencher os interstícios de ar. Cabe ressaltar que a sonda deve possuir uma relação entre o comprimento e o diâmetro (o comprimento deve ser no mínimo 20 vezes o diâmetro), garantindo que o fluxo de calor seja radial e unidirecional (Beck et al. ${ }^{[23]}$ ).

$\mathrm{Na}$ realidade, a fonte teórica linear é aproximada por uma resistência elétrica fina e o sólido infinito é substituído por uma amostra finita. Desta forma, percebe-se que o tempo mínimo utilizado para o cálculo da condutividade e da difusividade térmica fica limitado pela capacidade térmica do fio e pela resistência de contato entre o fio e a amostra, enquanto que o tempo máximo é limitado pelo tamanho finito da amostra. Assim, utiliza-se a região intermediária para o cálculo dessas propriedades térmicas no método do fio quente, pois esta apresenta o mesmo comportamento para as curvas experimental e numérica. Além deste cuidado, deve-se adotar alguns procedimentos para assegurar a confiabilidade e precisão dos resultados: utilizar uma resistência elétrica fina, que se aproxime o máximo possível da fonte de calor linear teórica; assegurar o melhor contato possível entre ambas as amostras e o fio quente, reduzindo assim os efeitos da resistência de contato; não considerar, para efeito de cálculo, o trecho inicial da curva, eliminando assim os efeitos de contato térmico entre a resistência elétrica e o material da amostra; e limitar o tempo de ensaio para assegurar que o tamanho finito da amostra não afete as temperaturas medidas.

\section{Descrição da bancada}

A bancada utilizada para determinar as propriedades do PVC e do Poliamida está representada pelas Figura 4 e 5 . O cilindro de PVC e Poliamida possuem as mesmas dimensões: 50,00 mm de diâmetro e 100,00 mm de comprimento. O fio que gera o calor possui resistência de $8,25 \Omega$ e as seguintes dimensões: $61,00 \mathrm{~mm} \mathrm{de}$ comprimento e $0,23 \mathrm{~mm}$ de diâmetro. Este fio é ligado a uma fonte de alimentação digital INSTRUTEMP ST - 305D, para fornecer a potência necessária. Os valores de corrente e tensão aplicados foram medidos pelos multímetros Instrutherm MD-380 e Minipa ET-2042C previamente calibrados para obter com precisão a potência gerada no interior da amostra. Devido à resistência de contato e presença de interstícios de ar entre a sonda, o fio de resistência e o termopar, foi necessário aplicar a pasta térmica de prata Arctic Silver 5. A grande vantagem desta pasta refere-se a sua alta condutividade térmica. Para ajudar a garantir um fluxo unidirecional e eliminar grande parte da convecção causada pela circulação do ar no ambiente,

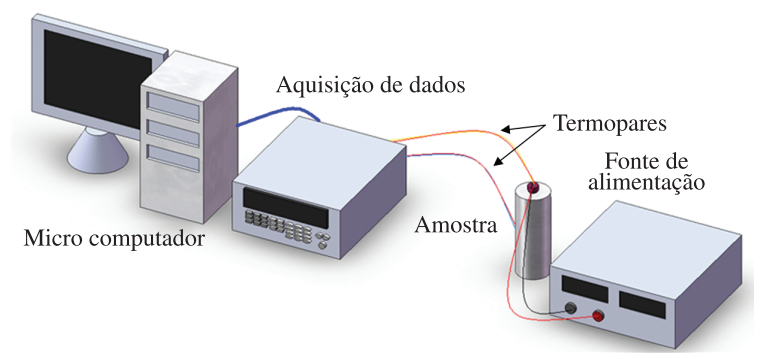

Figura 4. Esquema da bancada experimental utilizada. 
isolou-se a montagem com lã de vidro e borracha. As temperaturas foram coletadas através de termopares do tipo K (40AWG), para medir a temperatura internamente, e tipo $\mathrm{T}$ (30AWG) na porção exterior da amostra, ambos calibrados usando um banho calibrador de temperatura Marconi MA 184 com uma resolução de $\pm 0,01{ }^{\circ} \mathrm{C}$. Estes termopares foram conectados a aquisição de dados Agilent 34980A controlada por um micro computador. Visando obter melhores resultados, todos os experimentos foram realizados com a temperatura da sala controlada.

\section{Análise dos Resultados}

\section{Policloreto de vinila (pvc)}

Realizou-se 20 experimentos para a amostra de PVC. Cada experimento teve uma duração média de 160 segundos, sendo que os dados foram coletados em intervalos de 0,1 segundo. A potência gerada foi calculada através do valor de corrente aplicada, obtido pelo multímetro, e da resistência do fio, sendo respectivamente, $0,14 \mathrm{~A}$ e $8,25 \Omega$. O aquecimento foi imposto durante aproximadamente 60 segundos.

Deve-se ressaltar que, para realizar a determinação das propriedades, os dados coletados no início e no fim do experimento foram descartados conforme discutido anteriormente. Desta forma, apenas os pontos que se encontraram na faixa intermediária da curva foram utilizados. Esses pontos podem ser visualizados pela Figura 6, gráfico da temperatura em função do logaritmo neperiano do tempo, e constituem o intervalo de 2 a 4 segundos na escala logarítmica, o que equivale ao intervalo de 7 a 54 segundos do tempo total do experimento.

Os resultados médios obtidos para as propriedades do PVC são 0,157 para $k$ e $1,341 \times 10^{-6}$ para o $\alpha$, resultando em uma diferença de $0,641 \%$ e $1,745 \%$ respectivamente para $k$ e $\alpha$, quando comparado ao valores obtidos por Lima e Silva et al. ${ }^{[24]}$. Além disso, os valores de desvio padrão apresentados são de $\pm 0,002$ para $k$ e $\pm 0,018 \times 10^{-7}$ para $\alpha$.

Realizando uma análise apurada dos valores estimados pelos experimentos em questão, pode-se observar que o trabalho obteve êxito na determinação da condutividade e difusividade térmica do Policloreto de Vinila (PVC). Esta conclusão pôde ser estabelecida visto que se encontrou um baixo desvio padrão, uma diferença menor que $2 \%$ quando comparado ao valor fornecido pela literatura, e por fim, apresentou ótima repetibilidade, demonstrando que a bancada experimental é confiável. Além destas análises, efetuou-se a comparação destes resultados com os valores obtidos pela técnica apresentada por Tillmann et al. ${ }^{[25]}$. Esta comparação é justificável, pois as amostras utilizadas em ambos os trabalhos tiveram sua condutividade térmica medida pelo IPT $^{[26]}$. Sendo assim, confirmou-se a qualidade dos resultados obtidos pelo Método do Fio Quente, pois os resultados obtidos por ambas as técnicas apresentam uma diferença menor que $5 \%$.

\section{Poliamida}

Este tópico é destinado à análise dos resultados encontrados para a poliamida. 20 experimentos foram realizados de forma similar ao PVC. O tempo total do ensaio foi cerca de 160 segundos, porém somente no intervalo de 2 a 4 segundos, considerando a escala logarítmica, as propriedades foram estimadas. $O$ fluxo de calor foi gerado empregando-se uma corrente de $0,14 \mathrm{~A}$ ao fio de resistência de $8,25 \Omega$ e as temperaturas foram medidas no intervalo de 0,1 segundo. O fluxo gerado foi aplicado num intervalo de 90 segundos. A Figura 7 apresenta os pontos utilizados na determinação de $k$ e $\alpha$.

Os resultados médios obtidos para as propriedades da Poliamida são 0,305 para $k$ e $1,497 \times 10^{-7}$ para o $\alpha$, resultando em uma diferença de $0,993 \%$ e $1,629 \%$ respectivamente para $k$ e $\alpha$, quando comparado ao valores obtidos por Santos et al. ${ }^{[3]}$. Além disso, os valores de desvio padrão apresentados são de $\pm 0,004$ para $k$ e $\pm 0,047 \times 10^{-7}$ para $\alpha$.

De maneira semelhante ao PVC, os valores encontrados para a Poliamida apresentaram erros menores que $2 \%$ e pequenos desvios padrão quando comparados aos valores da literatura, conferindo à montagem experimental, confiabilidade e precisão na determinação das propriedades. Com o intuito de garantir maior confiabilidade ao resultado encontrado, utilizou-se o Método da Placa Quente Protegida $^{[5]}$ para obter o valor da condutividade térmica da poliamida. Novamente, obteve-se um resultado com diferença menor que 3\% quando comparado ao Método do Fio Quente.

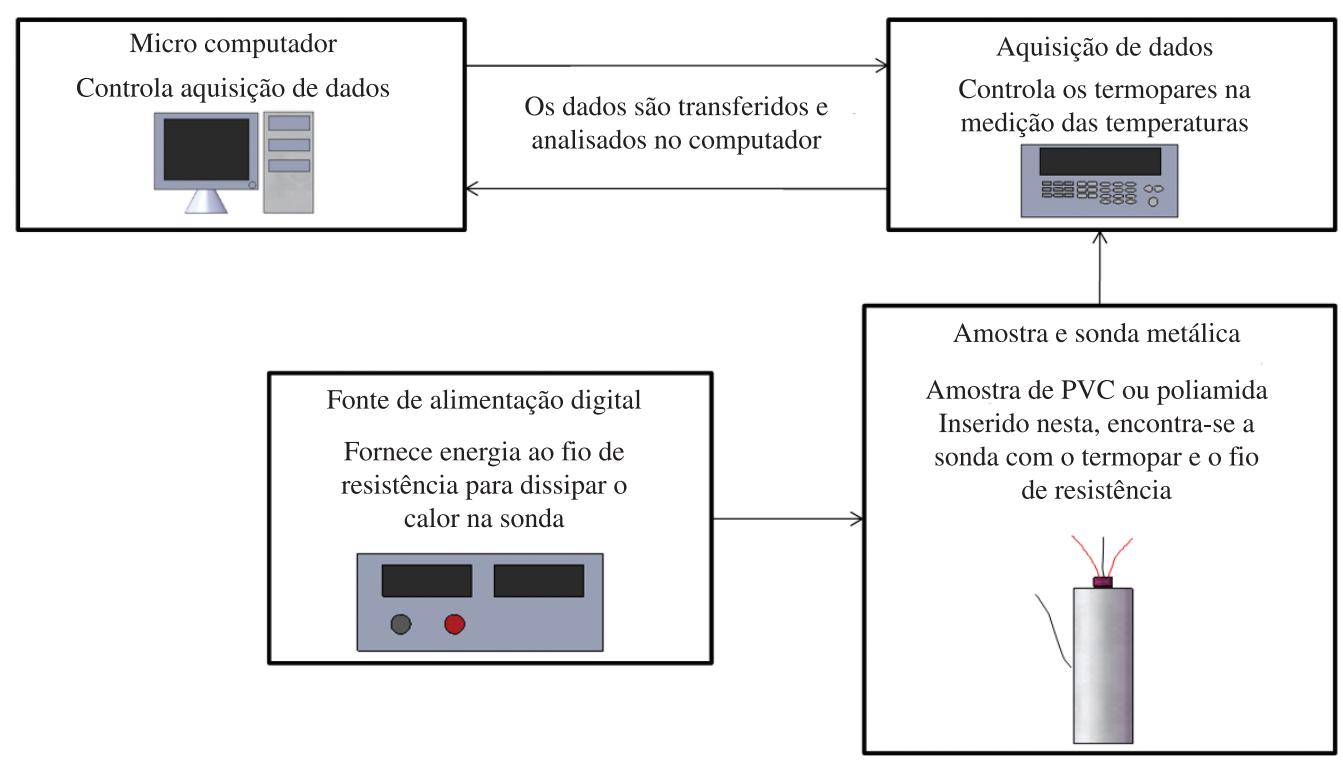

Figura 5. Bancada experimental em blocos ilustrativos. 


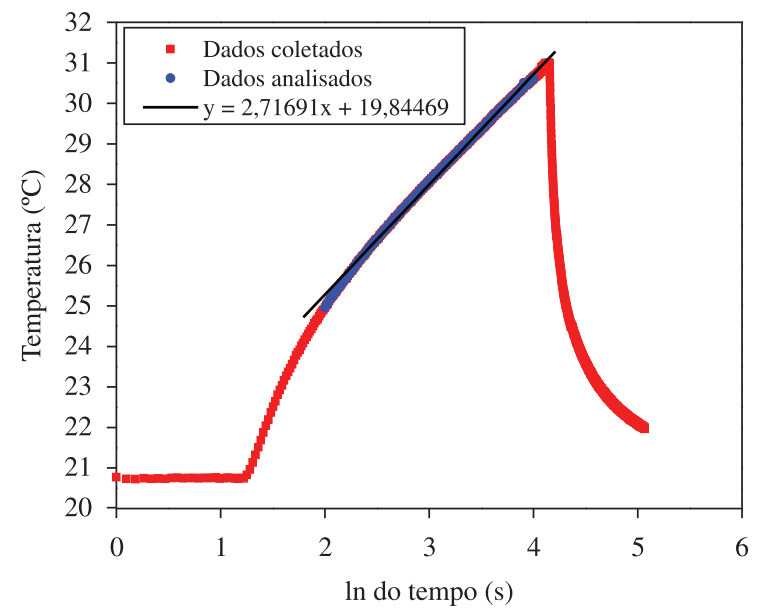

Figura 6. Distribuição de temperatura para o PVC.

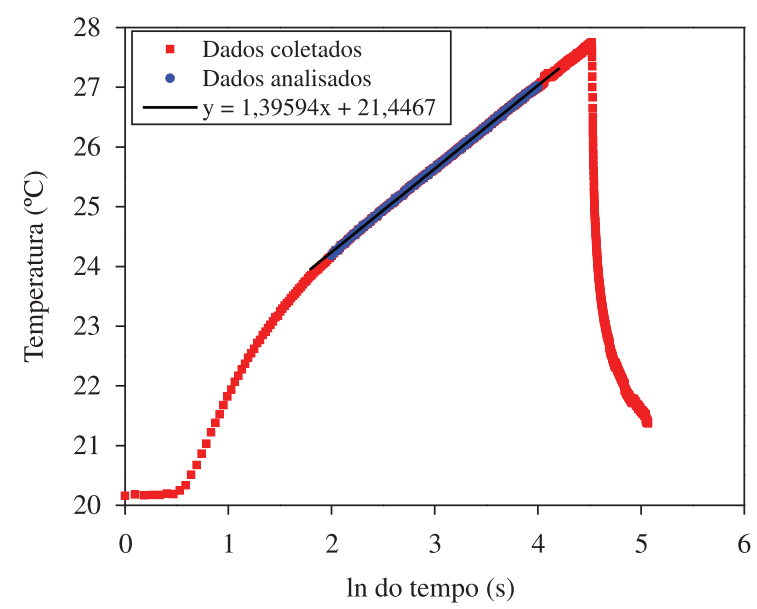

Figura 7. Distribuição de temperatura para a Poliamida.

\section{Conclusão}

Neste trabalho as propriedades termofísicas condutividade e a difusividade térmica do PVC e da Poliamida foram obtidas usando o Método do Fio Quente com uma sonda metálica diferenciada. Os valores obtidos para as propriedades estão de acordo com os valores disponíveis na literatura. Esta afirmação pode ser confirmada devido às diferenças entre os valores da literatura e os valores médios obtidos pelos experimentos apresentarem uma diferença menor que $2 \%$. Além disso, os resultados obtidos foram comparados a outras técnicas e apresentaram resultados satisfatórios. Um diferencial deste trabalho foi à utilização de uma sonda de dimensões reduzidas que apresentou resultados satisfatórios, visto que transferiu para a amostra praticamente todo o calor gerado, possibilitando uma maior precisão na determinação das propriedades. Além de permitir manusear o aparato com grande facilidade entre a realização dos experimentos, devido ao novo dispositivo de fixação dos componentes e a utilização de amostras menores.

\section{Agradecimentos}

Os autores agradecem ao CNPq, FAPEMIG e CAPES pelo suporte financeiro.

\section{Referências Bibliográficas}

1. Parker, W. J.; Jenkins, R. J.; Butler, C. P. \& Abbot, G. L. - J. Applied Phys., 32, p.1679-1684 (1961). http://dx.doi.org/10.1063/1.1728417

2. Eriksson, R., Hayashi, M. \& Seetharaman, S. -"Thermal Diffusivity Measurements of Liquid Silicate Melts", in: Proceedinfs of the $16^{\circ}$ European Conference on Thermophysical Properties - ECTP, London - UK (2002).

3. Santos, W. N.; Gregório Filho, R.; Mummery, P. E. \& Wallwork, A. - Polímeros, 14, p.354-359 (2004).

4. Associação Brasileira de Normas Técnicas - ABNT. - "NBR 15200-4: Medição da Resistência Térmica e da Condutividade Térmica pelo Princípio da Placa Quente Protegida", ABNT (2005).

5. Lima, G. M.; Carollo, L. F. S.; Lima e Silva, A. L. F.; Güths, S. \& Lima e Silva, S. M. M. - "Determinação da Condutividade pelo Método da Placa Quente Protegida", in: Anais do $5^{\circ}$ Congresso Nacional de Engenharia Mecânica - CONEM, Salvador - BA (2008).

6. Wulf, R., Barth, G. \& Gross, U. - "Intercomparison of Insulation Thermal Conductivities Measured by Various Methods", in: Proceedinfs of the $17^{\circ}$ European Conference on Thermophysical Properties - ECTP, Bratislava, Slovak Republic (2005).

7. Schieimacher, A. L. - Wiedemann Ann. Phys., 34, p.38 (1888).

8. Van Der Held, E. F. M. \& Van Drunen, F. G. - Physics, 15, p.865 (1949).

9. Blackwell, J. H. - J. Applied Phys., 25, p.137 (1954). http://dx.doi. org/10.1063/1.1721592

10. Haupin, W. E. - Am. Ceram. Soc. Bull., 39, p.139 (1960).

11. Fujii, M.; Zhang, X.; Fujiwara S.; Tomimura, T. \& Imaishi, N. - Rep. Inst. Advanc. Mater. Study Kyushu Univ., 8, p.99-105 (1994).

12. Zhang, X.; Fujii, M.; Gu, H. \& Xie, H. - Meas. Sci. Tech., 17, p.208 (2006). http://dx.doi.org/10.1088/0957-0233/17/1/032

13. Zhang, X.; Hendro, W.; Fujii, M.; Tomimura, T. \& Imaishi, N. - Int. J. Thermophysics, 23, p.1077 (2002). http://dx.doi. org/10.1023/A:1016394104244

14. Tomimura, T.; Maki, S.; Zhang X. \& Fujii, M. - Heat Transf. - Asian Res., 36, p.540 (2004). http://dx.doi.org/10.1002/htj.20032

15. Nahor, H. B.; Scheerlinck, N.; Van Impe, J. F. \& Nicolaï, B. M. J. Food Eng., 57, p.103 (2003). http://dx.doi.org/10.1016/ S0260-8774(02)00278-9

16. Adjali, M. H. \& Laurent, M. - Int. J. Heat and Mass Transf., 50, p.4623 (2007). http://dx.doi.org/10.1016/j.ijheatmasstransfer.2007.03.005

17. Yamasue, E.; Susa, M.; Fukuyama, H. \& Nagata, K. - "Deviation from Wiedemann-Franz Law for Thermal Conductivity of liquid Tin and Lead at Elevated Temperature", Kyoto University, Yoshida-Honmachi, Sakyo-ku, Kyoto, p.606 (1999).

18. Cheng, S. \& Xie, H. - Meas. Sci. Tech., 12, p.58-62 (2001). http:// dx.doi.org/10.1088/0957-0233/12/1/307

19. Zhang X.; Wicaksono, H.; Fujiwara, S. \& Fujii, M. - High Temp. - High Pres., 34, p.617-625 (2002).

20. American Society for Testing and Materials - ASTM. - "D5334-08: Standard Test Method for Determination of Thermal Conductivity of Soil and Soft Rock by Thermal Needle Probe Procedure", ASTM (2009).

21. Özisik, M. N. - "Heat Conduction", John Wiley \& Sons, New York (1993).

22. Vanderplaats, G. N. - "Numerical Optimization Techniques for Engineering Design", McGraw Hill, New York (2005).

23. Beck, J. V.; Cole, K. D.; Haji-Sheikh, A. \& Litkouhi, B. - "Heat Conduction Using Green's Functions", Hemisphere Publishing Corporation, Washington (1992). 
24. Lima e Silva, S. M. M.; Ong, T. H. \& Guimarães, G. - J. Braz. Soc. Mech. Sci. Eng., 25, p.9 (2003). http://dx.doi.org/10.1590/ S1678-58782003000100002

25. Tillman, A. R.; Borges, V. L.; Guimarães, G.; Lima e Silva, A. L. F. \& Lima e Silva, S. M. M. - J. Braz. Soc. Mech. Sci. Eng., 30, p.269 (2008). http://dx.doi.org/10.1590/S1678-58782008000400001
26. Instituto de Pesquisas Tecnológicas - IPT. - "Determinação de Condutividade Térmica", IPT (2004). Relatório de Ensaio nº 908637.

Enviado: $21 / 10 / 10$ Reenviado: $23 / 05 / 11$ Aceito: 05/09/11 